\title{
MIMO-OFDM CHANNEL ESTIMATION A Pilot Sequence Design for Time-domain Processing
}

\author{
Carlos Ribeiro \\ Instituto de Telecomunicações, Universidade de Aveiro, Aveiro, Portugal \\ Instituto Politécnico de Leiria, Morro do Lena - Alto Vieiro, Leiria, Portugal \\ cribeiro@estg.ipleiria.pt \\ Atílio Gameiro \\ Instituto de Telecomunicações, Universidade de Aveiro, Aveiro, Portugal \\ amg@det.ua.pt
}

Keywords: OFDM, MIMO, pilot-aided channel estimation, pilot sequence design, time-domain processing.

\begin{abstract}
A pilot sequence design for MIMO-OFDM systems is investigated where all transmit antennas share the same sub-carriers to convey pilot symbols. The pilot sequences are endowed with phase-shifting properties, granting the receiver the possibility of extracting the impulse responses for all channels directly from the antennas' time-domain received signals, without co-channel interference. A linear processing of the timedomain samples is all that is needed to get the channel impulse responses, resulting in an algorithm with very low computational load. The feasibility of the investigated method is substantiated by system simulation using indoor and outdoor broadband wireless channel models.
\end{abstract}

\section{INTRODUCTION}

Future mobile broadband applications will require reliable high data-rate wireless communication systems. In recent years, multiple-input multipleoutput orthogonal frequency division multiplexing (MIMO-OFDM) transmission systems (Nee, 2000), (Stuber, 2004), (Sampath, 2005), (Paulraj, 2004), emerged as the scheme with the potential to fulfill these conditions, with bandwidth efficiency and robustness to frequency selective channels, common in mobile personal communication systems.

The accurate extraction of the channel state information is crucial to realize the full potential of the MIMO-OFDM system. The performance of the channel estimator is vital for diversity combining, coherent detection and decoding, and resource allocation operations. The co-channel interference inherent to the system, where the received signal is the superposition of the signals transmitted simultaneous from the different antennas, puts an additional challenge on the design of the channel estimation algorithm.

A decision-directed channel estimation scheme that attempted to minimize the co-channel interference was published in $(\mathrm{Li}, 1999)$. The proposed algorithm exhibits a high computational load. A simplified and enhanced algorithm, introducing a data-aided scheme for the data transmission mode, is presented in $(\mathrm{Li}, 2002)$. The topic attracted a significant attention and has been the focus of investigation in multiple publications (Shin, 2004), (Zhang, 2005), (Zamiri-Jafarian, 2007) and references therein.

The design of training symbols and pilot sequences with the ability to decouple the cochannel interference and minimize the channel estimation mean square error (MSE) for MIMOOFDM was addressed in several publications $(\mathrm{Li}$, 2002), (Barhumi, 2003) and (Minn, 2004).

Most publications on the topic of training-signal or pilot-aided channel estimation use the frequencydomain (FD) least squares (LS) estimates as the starting point for the analysis of the estimation algorithm or the design of the training sequence. It was established in (Ribeiro, 2007) that in singleinput single-output (SISO) OFDM a time-domain (TD) equivalent LS estimate could be obtained using a simple linear operation on the received signal, if the used pilot sequence fulfills certain conditions (training sequences were treated as particular pilot sequences with no data symbols in-between consecutive pilot sub-carriers). 
In this paper we develop a MIMO-OFDM pilot sequence design and associated channel estimation method that succeeds in extracting each channel impulse response (CIR) estimate with minimal computational load. The investigated scheme can be used in either spatial multiplexing or space-time coded systems, with all transmit antennas sharing the same sub-carriers to convey its individual pilot sequences, thus minimizing the system's pilot overhead. Using different phase-shifting pilot sequences for each transmit antenna, together with the result in (Ribeiro, 2007), the method succeeds in separating the overlapping FD received pilots sequences by using a TD algorithm that successfully estimates the different CIRs and eliminates the cochannel interference, under given conditions. The STC algorithm (Li, 1999) or the TD LMMSE criterion (Edfors, 1996) can be incorporated to improve the estimate at little or no added computational cost.

The paper is organized as follows. Next Section gives a brief introduction to the wireless multipath channel and the MIMO-OFDM baseband model. In Section 3 the investigated pilot sequence design is developed. The feasibility of the new method is substantiated by simulation results presented in Section 4. Finally, conclusions are drawn in Section 5 .

\section{MIMO-OFDM IN MOBILE WIRELESS CHANNELS}

Before introducing the investigated method, we will briefly overview the mobile wireless multipath channel and the considered MIMO-OFDM baseband model.

Throughout the text, the notation $(\sim)$ is used for TD vectors and elements and its absence denotes FD vectors and elements. The index $n$ denotes TD elements and $k$ FD elements. Unless stated otherwise, the vectors involved in the transmission/reception process are column vectors with $N_{C}$ complex elements. The superscripts $(.)^{T}$ and $(.)^{H}$ denote transpose and Hermitian transpose, respectively.

\subsection{The Wireless Multipath Channel}

Let's consider that the system transmits over multipath Rayleigh fading wireless channels, modeled by the discrete-time CIR,

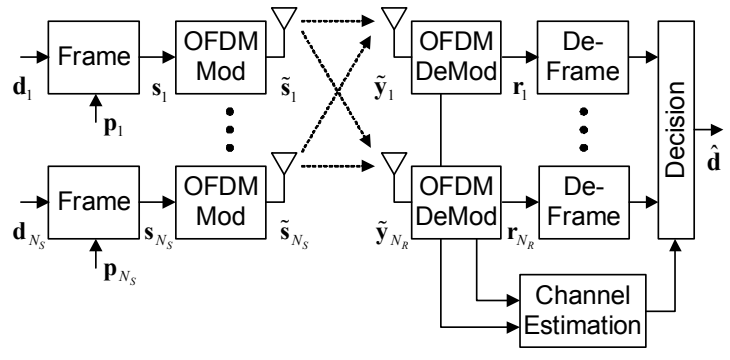

Figure 1: MIMO-OFDM baseband system model.

$$
\tilde{h}[n]=\sum_{l=0}^{L-1} \alpha_{l} \delta\left[n-\tau_{l}\right],
$$

where $L$ is the number of channel paths, $\alpha_{l}$ and $\tau_{l}$ are the complex value and delay of path $l$, respectively. The paths are assumed to be statistically independent, with normalized average power, $\sum_{l=0}^{L-1} \sigma_{l}^{2}=1$, where $\sigma_{l}^{2}$ is the average power of path $l$. The channel is time-variant due to the motion of the mobile terminal (MT), but we will assume that the CIR is constant during one OFDM symbol. The time dependence of the CIR is not present in the notation for simplicity. Assuming that the insertion of a long enough cyclic prefix (CP) in the transmitter assures that the orthogonality of the sub-carriers is maintained after transmission, the channel frequency response (CFR) can be expressed as,

$$
h[k]=\sum_{l=0}^{L-1} \alpha_{l} \exp \left[-j \frac{2 \pi}{N_{C}} k l\right],
$$

where $N_{C}$ is the total number of sub-carriers of the OFDM system.

\subsection{MIMO-OFDM Baseband Model}

Let's consider the MIMO-OFDM baseband system with $n_{S}$ transmit antennas and $n_{R}$ receive antennas depicted in Figure 1. The system uses $N_{C} M$-ary PSK or QAM modulated sub-carriers. At time $n$, the binary data vector $\mathbf{b}$ is coded into $n_{S}$ modulating vectors $\left\{\mathbf{d}_{s}: s=1, \ldots, n_{S}\right\}$. The transmit antennas simultaneously send $n_{S}$ OFDM signals modulated by $\mathbf{d}_{s}$. 
To assist in the channel estimation process, pilot symbols are multiplexed together with data symbols. Each antenna path uses a different FD pilot vector $\mathbf{p}_{s}$. Vectors $\mathbf{d}_{s}$ and $\mathbf{p}_{s}$ contain non-zero values at disjoint positions (sub-carriers). The resulting FD signal transmitted by antenna $s$ is $\mathbf{s}_{s}=\mathbf{d}_{s}+\mathbf{p}_{s}$.

The OFDM modulator present on each antenna path performs an $N_{C}$-points inverse discrete Fourier transform (IDFT) operation to transform the vector $\mathbf{s}_{s}$ to TD. An $L$ samples long guard interval, in the form of $\mathrm{CP}$, is prefixed to the resulting vector. Antenna $s$ TD transmitted vector $\tilde{\mathbf{s}}_{s}$ is,

$$
\tilde{\mathbf{s}}_{s}=\mathbf{A}_{C P} \mathbf{F}^{H} \mathbf{s}_{s}=\mathbf{A}_{C P}\left(\tilde{\mathbf{d}}_{s}+\tilde{\mathbf{p}}_{s}\right),
$$

where $\quad \mathbf{F} \triangleq\left(e^{-j 2 \pi k n / N_{C}}\right)_{k, n=0,0}^{N_{C}-1, N_{C}-1}$ is the $N_{C} \times N_{C}$ discrete Fourier transform (DFT) matrix and $\mathbf{A}_{C P}=\left[\begin{array}{ll}\mathbf{I}_{N_{C} \times L} & \mathbf{I}_{N_{C}}\end{array}\right]^{T}$ is the matrix that adds the CP, with $\mathbf{I}_{N_{C}}$ denoting the $N_{C} \times N_{C}$ identity matrix and $\mathbf{I}_{N_{C} \times L}$ denoting the last $L$ columns of $\mathbf{I}_{N_{C}}$. The TD vectors $\tilde{\mathbf{d}}_{s}$ and $\tilde{\mathbf{p}}_{s}$ collect, respectively, the components of data symbols and pilot symbols present in $\tilde{\mathbf{s}}_{s}$.

The transmission over uncorrelated wireless channels results in a received signal vector $\tilde{\mathbf{y}}$ at receive antenna $r$ consisting of $n_{S}$ superimposed transmitted signal samples,

$$
\tilde{\mathbf{y}}_{r}=\sum_{s=0}^{n_{S}-1} \tilde{\mathbf{y}}_{r, s}+\tilde{\mathbf{w}}^{\prime}=\sum_{s=0}^{n_{S}-1} \tilde{\mathbf{H}}_{r, s}^{\prime} \tilde{\mathbf{s}}_{s}+\tilde{\mathbf{w}}^{\prime}
$$

where the vector $\tilde{\mathbf{w}}^{\prime}$ is made-up of independent and identically distributed (iid) zero mean additive white Gaussian noise (AWGN) samples with variance $\sigma_{\mathbf{w}}^{2}$. The vector $\tilde{\mathbf{y}}_{r, s}=\tilde{\mathbf{H}}_{r, s}{ }_{r, s} \tilde{\mathbf{s}}_{s}$ represents the contribution from transmit antenna $s$ to the received signal; $\quad \tilde{\mathbf{H}}_{r, s}^{\prime}$ is the $\left(N_{C}+L\right) \times\left(N_{C}+L\right)$ lower triangular Toeplitz channel convolution matrix with first column $\left[\begin{array}{ll}\tilde{\mathbf{h}}_{r, s}^{T} & \mathbf{0}_{1 \times N_{C}}\end{array}\right]^{T}$, where $\tilde{\mathbf{h}}_{r, s}$ is the column $L$-vector with the discrete-time CIR from transmit antenna $s$ to receive antenna $r$ and $\mathbf{0}_{1 \times N_{C}}$ is a null $N_{C}$-vector.
With the assumption that the signals in the receive antennas are mutually uncorrelated, the channel estimation at the receiver will be independent for all channels and, in the following, the receive antenna index will be dropped for notation simplicity.

The OFDM demodulator in each receiving antenna path starts by removing the $\mathrm{CP}$ from each symbol. The resulting vector for symbols with pilots and data is,

$$
\begin{aligned}
\tilde{\mathbf{r}} & =\mathbf{R}_{C P} \tilde{\mathbf{y}}=\sum_{s=0}^{n_{S}-1} \mathbf{R}_{C P} \tilde{\mathbf{H}}^{\prime}{ }_{s} \mathbf{A}_{C P}\left(\tilde{\mathbf{d}}_{s}+\tilde{\mathbf{p}}_{s}\right)+\mathbf{R}_{C P} \tilde{\mathbf{w}}^{\prime} \\
& =\sum_{s=0}^{n_{S}-1} \tilde{\mathbf{H}}_{s}\left(\tilde{\mathbf{d}}_{s}+\tilde{\mathbf{p}}_{s}\right)+\tilde{\mathbf{w}}=\sum_{s=0}^{n_{S}-1} \tilde{\mathbf{r}}_{s}+\tilde{\mathbf{w}}
\end{aligned}
$$

where $\mathbf{R}_{C P}=\left[\begin{array}{ll}\mathbf{0}_{N_{C} \times L} & \mathbf{I}_{N_{C}}\end{array}\right]$ is the matrix that removes the CP, $\tilde{\mathbf{w}}=\mathbf{R}_{C P} \tilde{\mathbf{w}}^{\prime}$ is the TD noise vector, $\tilde{\mathbf{r}}_{s}=\tilde{\mathbf{H}}_{s}\left(\tilde{\mathbf{d}}_{s}+\tilde{\mathbf{p}}_{s}\right)$ represents the contribution from transmit antenna $s$ to the received vector and $\tilde{\mathbf{H}}_{s}=\mathbf{R}_{C P} \tilde{\mathbf{H}}_{s}^{\prime} \mathbf{A}_{C P}$ is the $N_{C} \times N_{C}$ circulant matrix for channel $s$, with circulant vector $\left[\begin{array}{ll}\tilde{\mathbf{h}}_{s}^{T} & \mathbf{0}_{1 \times\left(N_{C}-L\right)}\end{array}\right]^{T}$.

The symbols are transformed back to FD with a DFT operation. The resulting FD column $N_{C}$-vector can be expressed as,

$$
\mathbf{r}=\mathbf{F} \tilde{\mathbf{r}}=\sum_{s=0}^{n_{s}-1} \mathbf{H}_{s}\left(\mathbf{d}_{s}+\mathbf{p}_{s}\right)+\mathbf{w},
$$

where $\mathbf{H}_{s}$ is the $N_{C} \times N_{C}$ diagonal matrix whose diagonal elements are defined by (2) and $\mathbf{w}$ is the FD noise vector.

The deframing block in each receive antenna path separates the signals in the sub-carriers conveying pilots and data symbols. The signals in the data sub-carriers are fed to the decision block.

\section{A PILOT SEQUENCE DESIGN FOR TD PROCESSING}

The channel estimation algorithm must estimate the $n_{S} n_{R}$ channels from the receive antennas' signals. The proposed method estimates the channels from the symbols carrying pilots and data (the training sequence is a particular case and the results can be easily extended to it). All the processing required to estimate the CIR is performed immediately on the 
TD received vector $\tilde{\mathbf{r}}$, eliminating the need to go from TD to FD and back to TD to finally obtain the CIR estimate (Li, 1999), (Li, 2002). The present algorithm has a very low computational load and no performance trade-offs. Under given conditions, the co-channel interference is entirely eliminated. Dataaided channel estimation algorithms ( $\mathrm{Li}, 2002)$ can be incorporated to further improve the algorithm's performance.

Consider the set of sub-carriers $\wp$ dedicated to convey pilot symbols,

$$
\wp=\left\{0, N_{f}, 2 N_{f}, \cdots, N_{C}-N_{f}\right\},
$$

where the pilot distance $N_{f}$ can range from 1 (particular case where all sub-carriers in the OFDM symbol are dedicated to transmit pilots - training symbol) to $N_{C}$, fulfilling the condition $\frac{N_{C}}{N_{f}}=N_{t} \in \mathbb{N}$. The set $\wp$ is common to all transmit antennas (pilot sub-carriers overlap in receive antennas).

The system uses distinct phase-shifted pilot sequences in each transmit antenna. The $k$-th element of the column $N_{C}$-vector $\mathbf{p}_{s}$ is defined by,

$$
p_{s}[k]=\sum_{m=0}^{N_{t}-1} \delta\left[k-m N_{f}\right] \exp \left[-j 2 \pi \frac{s}{n_{S}} m\right] \text {, }
$$

giving rise to the corresponding TD vector $\tilde{\mathbf{p}}_{s}$, whose $n$-th element is,

$$
\begin{aligned}
\tilde{p}_{s}[n] & =\frac{1}{N_{C}} \sum_{k=0}^{N_{C}-1} \sum_{m=0}^{N_{t}-1} \delta\left[k-m N_{f}\right] e^{-j 2 \pi \frac{s}{n_{S}} m} e^{j \frac{2 \pi}{N c} k n} \\
& =\frac{1}{N_{f}} \sum_{m=0}^{N_{f}-1} \delta\left[n-\frac{s}{n_{S}} N_{t}-m N_{t}\right], \frac{N t}{n_{S}} \in \mathbb{N}
\end{aligned}
$$

or equivalently,

$$
\left\{\begin{array}{l}
\tilde{p}_{o}[n]=\frac{1}{N_{f}} \sum_{m=0}^{N_{f}-1} \delta\left[n-m N_{t}\right] \\
\tilde{p}_{1}[n]=\frac{1}{N_{f}} \sum_{m=0}^{N_{f}-1} \delta\left[n-\frac{1}{n_{S}} N_{t}-m N_{t}\right] \\
\vdots \\
\tilde{p}_{n_{S}-1}[n]=\frac{1}{N_{f}} \sum_{m=0}^{N_{f}-1} \delta\left[n-\frac{n_{S}-1}{n_{S}} N_{t}-m N_{t}\right]
\end{array}\right.
$$

Equation (10) shows that the transmit antennas' pilot sequences do not overlap in TD, allowing the separation of each transmit antenna's CIR, as it will be demonstrated.

The $n$-th element of $\tilde{\mathbf{d}}_{s}$ can be expressed by,

$$
\tilde{d}_{s}[n]=\frac{1}{N_{C}} \sum_{\substack{k=0 \\ k \notin \wp}}^{N_{C}-1} d_{s}[k] e^{j 2 \pi \frac{k n}{N_{C}}},
$$

where $d_{s}[k]$ is the $k$-th element of $\mathbf{d}_{s}$ (complex data symbol conveyed by the $k$-th sub-carrier).

Replacing equation (9) in equation (5), the $n$-th element of $\tilde{\mathbf{r}}$ is,

$$
\begin{aligned}
\tilde{r}[n] & =\sum_{s=0}^{n_{S}-1} \tilde{r}_{s}[n]+\tilde{w}[n]=\sum_{s=0}^{n_{S}-1} \sum_{l=0}^{L-1} \tilde{h}_{s}[l] \tilde{d}_{s}[n-l] \\
& +\frac{1}{N_{f}} \sum_{s=0}^{n_{S}-1} \sum_{m=0}^{N_{f}-1} \tilde{h}_{s}\left[n-\frac{s}{n_{S}} N_{t}-m N_{t}\right]+\tilde{w}[n] \\
= & \sum_{s=0}^{n_{S}-1} \sum_{l=0}^{L-1} \tilde{h}_{s}[l] \tilde{d}_{s}[n-l]+\frac{1}{N_{f}} \sum_{m=0}^{N_{f}-1} \tilde{h}_{0}\left[n-m N_{t}\right] \\
& +\frac{1}{N_{f}} \sum_{m=0}^{N_{f}-1} \tilde{h}_{1}\left[n-\frac{1}{n_{S}} N_{t}-m N_{t}\right]+\cdots \\
& +\frac{1}{N_{f}} \sum_{m=0}^{N_{f}-1} \tilde{h}_{n_{S}-1}\left[n-\frac{n_{S}-1}{n_{S}} N_{t}-m N_{t}\right]+\tilde{w}[n]
\end{aligned}
$$

Equation (12) puts in evidence that antenna $r$ received signal is the sum of three distinct components: the data vectors $\tilde{\mathbf{d}}_{s}$ and pilot vectors $\tilde{\mathbf{p}}_{s}$, transmitted over its channels, and the AWGN. Looking carefully at the component dependent on the pilot vectors, it becomes clear that it is made-up of $N_{f}$ scaled replicas of each of the $n_{S}$ CIR, corresponding to the $n_{S}$ channels that link the transmit antennas with the considered receive antenna. Moreover, the replicas of each CIR are separated by $N_{t}$ samples and transmit antenna $s$ CIR replicas are time-shifted $N_{t} \frac{s}{n_{S}}$ samples from the reference position $m N_{t}, m \in\left\{0, \cdots, N_{f}-1\right\}$.

Figure 2 shows an example of a TD received vector $\tilde{\mathbf{r}}$ when $n_{S}=4$ antennas transmit one symbol carrying only pilots (data sub-carriers are not loaded) over independent BRAN-A model multipath channels (ETSI, 2001). The top plot represents the overlapping of the signals from all transmit antennas 
in one receive antenna. The four lower plots show how the CIR replicas corresponding to each transmit antenna are phase-shifted and can be separated from the overall signal. It gives a clear image of the condition that each CIR must fulfill so that consecutive replicas do not overlap.

Given the transmit antenna $s$ maximum channel delay spread, $\tau_{s}$, and the system's sampling interval, $\Delta t$, the minimum pilot distance that can be used without overlap of adjacent CIR replicas, thus enabling the best performance of the channel estimator, can be expressed as

$$
N_{f} \leq \frac{N_{C} \Delta t}{n_{S} \tau_{s}}
$$

As it will be demonstrated, if the condition in (13) is verified the co-channel interference will be eliminated. Otherwise, the overlapping of consecutive replicas will cause distortion in the estimation process and impose an MSE floor on the estimate.

Considering that the condition presented by (13) is fulfilled, the transmit antenna $s$ CIR estimate vector $\hat{\tilde{\mathbf{h}}}_{s}$ can be obtained immediately from the TD received vector $\tilde{\mathbf{r}}$ (Ribeiro, 2007),

$$
\begin{aligned}
& \hat{\tilde{h}}_{s}[n]=\left\{\begin{array}{l}
\sum_{m=0}^{N_{f}-1} \tilde{r}\left[n+\frac{s}{n_{S}} N_{t}-m N_{t}\right], n=0,1, \cdots, \frac{N_{t}}{n_{S}}-1 \\
0, \text { remaining }
\end{array}\right. \\
& =\left(u[n]-u\left[n-\frac{N_{t}}{n_{S}}+1\right]\right) \sum_{m=0}^{N_{f}-1} \tilde{r}\left[n+\frac{s}{n_{S}} N_{t}-m N_{t}\right] \\
& =\left(u[n]-u\left[n-\frac{N_{t}}{n_{S}}+1\right]\right) \times \\
& \left(\begin{array}{l}
\sum_{m=0}^{N_{f}-1} \sum_{s=0}^{n_{n^{-}-1}} \sum_{l=0}^{N_{f}-1} \frac{\tilde{h}_{s}\left[n-m N_{t}-l N_{t}\right]}{N_{f}}+\sum_{m=0}^{N_{f}-1} \tilde{w}\left[n+\frac{s N_{t}}{n_{S}}-m N_{t}\right] \\
+\frac{1}{N_{f}} \sum_{m=0}^{N_{f}-1} \sum_{s=0}^{n_{S}-1} \sum_{l=0}^{L-1} \tilde{h_{s}}[l] \tilde{d}_{s}\left[n+\frac{s}{n_{S}} N_{t}-m N_{t}-l\right]
\end{array}\right) \\
& =\left\{\begin{array}{l}
\tilde{h_{s}}[n]+\sum_{m=0}^{N_{f}-1} \tilde{w}\left[n+\frac{s}{n_{S}} N_{t}-m N_{t}\right], n=0,1, \cdots, \frac{N_{t}}{n_{S}}-1 \\
0, \quad \text { remaining }
\end{array},\right.
\end{aligned}
$$

where $u[n]$ is the unit step function. The operation in (14) estimates all $n_{S}$ CIR and must be repeated for all $n_{R}$ receive antennas. The result in (14) is possible because the data dependent component was eliminated, considering that (Ribeiro, 2007),

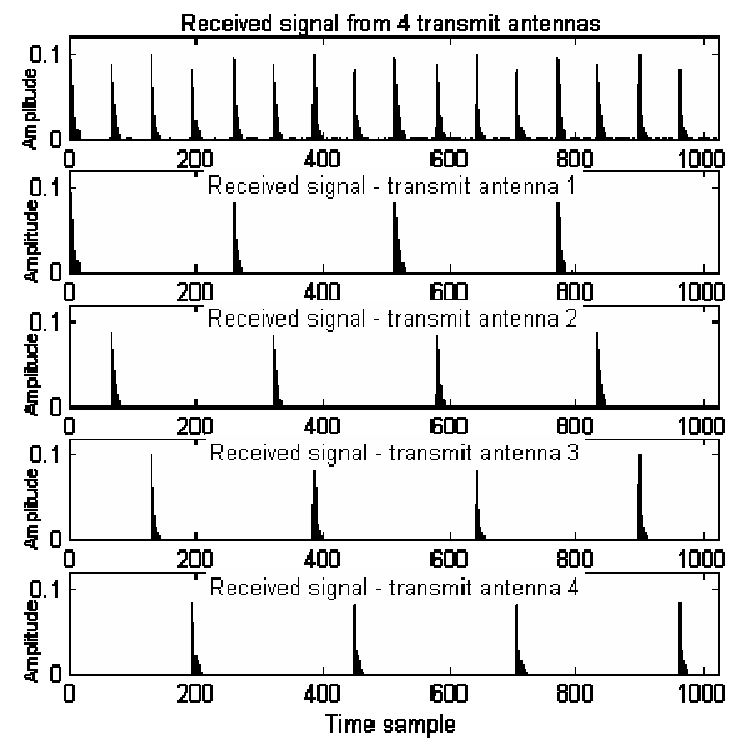

Figure 2: Decomposition of the combined received symbol (pilots only) for $n_{s}=4$ transmit antennas.

$$
\begin{aligned}
& \frac{1}{N_{f}} \sum_{m=0}^{N_{f}-1} \sum_{s=0}^{n_{S}-1} \sum_{l=0}^{L-1} \tilde{h}_{s}[l] \tilde{d}_{s}\left[n+\frac{s}{n_{S}} N_{t}-m N_{t}-l\right]= \\
& =\frac{1}{N_{C} N_{f}} \sum_{s=0}^{n_{S}-1} \sum_{l=0}^{L-1} \tilde{h}_{s}[l] \sum_{\substack{k=0 \\
k \notin \mathcal{S}}}^{N_{C}-1} d_{s}[k] \exp \left[j \frac{2 \pi}{N_{C}} k\left(n+\frac{s N_{t}}{n_{S}}-l\right)\right] \\
& \times \sum_{m=0}^{N_{f}-1} \exp \left[-j \frac{2 \pi}{N_{C}} k m N_{t}\right]=0
\end{aligned}
$$

The CIR estimate resulting of (14) is the TD LS equivalent of a SISO OFDM system (Ribeiro, 2007). Consider a constant envelope modulation (though it can be easily extended for any modulation) with transmit power equally distributed among the $n_{S}$ antennas, $E\left\{\left|s_{s}[k]\right|^{2}\right\}=1 / n_{S}$. Pilot and data subcarriers are transmitted with equal power. Assuming that the noise in the TD samples is iid, the noise variance in (14) is $\sigma_{w}^{2} / n_{S}$, resulting in the channel estimation MSE,

$$
M S E_{I N I}=N_{f} \sigma_{\mathbf{w}}^{2}
$$

This initial estimate can be improved by incorporating other TD algorithms at little or no added computational load. With the knowledge that the CIR energy is concentrated in a small set of taps, the significant tap catching (STC) scheme (Li, 1999) lowers the channel estimation MSE considerably. Further improvement is provided by the TD linear 
minimum MSE (LMMSE) filter (Edfors, 1996), if the channel correlation and noise variance are known a-priori (due to the CIR energy concentration, the estimation of these parameter is quite simpler in TD, when compared to FD).

Considering the more realistic approach of nonsample-spaced channel (where channel paths are not separated by multiples of $\Delta t$ and energy leakage occurs), other algorithms (Edfors, 1996) can provide further improvement with added computational load.

\subsection{Including the STC Algorithm}

The CIR energy of wireless multipath channels is commonly limited to a small set of taps. By identifying this set of significant taps and ignoring the remaining, we are removing noise from the channel estimate, thus improving it ( $\mathrm{Li}, 2002)$. Considering the channel model introduced in Section 2, the CIR will only have $L$ significant taps. Assuming that the set of significant taps is $\{L\},(14)$ can be rewritten

$$
\begin{aligned}
\hat{\tilde{h}}_{s}[n] & =\left\{\begin{array}{l}
\sum_{m=0}^{N_{f}-1} \tilde{r}\left[n+\frac{s}{n_{S}} N_{t}-m N_{t}\right], n \in\{L\} \\
0, \text { remaining }
\end{array}\right. \\
& =\left\{\begin{array}{l}
\tilde{h}_{s}[n]+\sum_{m=0}^{N_{f}-1} \tilde{w}\left[n+\frac{s}{n_{S}} N_{t}-m N_{t}\right], n \in\{L\} \\
0, \text { remaining }
\end{array}\right.
\end{aligned}
$$

By eliminating the noise in the non-significant taps, the noise variance in (17) is reduced to $\sigma_{\mathrm{w}}^{2} \frac{L N_{f}}{N_{C}}$ and the resulting channel estimation MSE is,

$$
M S E_{S T C}=\sigma_{\mathrm{w}}^{2} \frac{n_{S} L N_{f}^{2}}{N_{C}}=M S E_{I N I} \frac{n_{S} L N_{f}}{N_{C}} .
$$

This MSE tends to the initial MSE when $L$ goes to $\frac{N_{C}}{n_{S} N_{f}}$ (limiting condition in (13)). The channel estimation MSE achieved in ( $\mathrm{Li}, 2002)$ is the same as in (18) when $N_{f}=1$ (training sequence).

The use of the STC algorithm actually lowers the computational load by limiting the summation interval. An error floor will limit the performance for high values of SNR if the set $\{L\}$ is not properly estimated and some CIR energy is removed.

\subsection{Including TD LMMSE Filter}

For low values of SNR, a significant improvement in the channel estimation can be achieved by using the TD LMMSE filter that minimizes the effect of the noise in the estimate.

Considering the channel model introduced in Section 2, where all channel taps are separated by multiples of $\Delta t$, the TD MMSE filter is implemented by a diagonal matrix (Edfors, 1996) whose diagonal elements are defined by $\frac{R_{\tilde{h} \tilde{h}}[n]}{R_{\tilde{h} \tilde{h}}[n]+\sigma_{w}^{2} / N_{f}}$, where $\mathbf{R}_{\tilde{h} \tilde{h}}$ is the CIR correlation matrix. Because all paths of all channels are uncorrelated, the matrix will only have $L$ non-zero values in the diagonal elements defined by $\{L\}$. Therefore, the operation can be implemented simultaneously with the operation in (13),

$$
\begin{aligned}
\hat{\tilde{h}}_{s}[n] & =\left\{\begin{array}{l}
\frac{R_{\tilde{h} \tilde{h}}[n]}{R_{\tilde{h} \tilde{h}}[n]+\sigma_{w}^{2}} \sum_{m=0}^{N_{f}-1} \tilde{r}\left[n+\frac{s}{n_{S}} N_{t}-m N_{t}\right], n \in\{L\} \\
0, \text { remaining }
\end{array}\right. \\
& =\left\{\begin{array}{l}
\frac{R_{\tilde{h} \tilde{h}}[n]\left(\tilde{h}_{s}[n]+\sum_{m=0}^{N_{f}-1} \tilde{w}\left[n+\frac{s}{n_{S}} N_{t}-m N_{t}\right]\right)}{R_{\tilde{h} \tilde{h}}[n]+\sigma_{w}^{2}}, n \in\{L\} \\
0, \text { remaining }
\end{array}\right.
\end{aligned}
$$

The resulting channel estimation MSE (Edfors, 1996) is,

$$
M S E_{L M M S E}=\sigma_{\mathbf{w}}^{2} \frac{n_{S} N_{f}^{2}}{N_{C}} \sum_{l \in\{L\}} \frac{R_{\tilde{h} h}[l]}{R_{\tilde{h} h}[l]+\sigma_{\mathbf{w}}^{2} \frac{n_{S} L N_{f}^{2}}{N_{C}}}
$$

Observing (20), we can conclude that when $S N R \rightarrow \infty, M S E_{\text {LMMSE }} \rightarrow M S E_{S T C}$.

The use of LMMSE filter lowers the computational load by limiting the summation interval but requires additional calculations to estimate the channel correlation and noise variance.

For non-sample-spaced channels, the CIR correlation is no longer a diagonal matrix and this estimator no longer guaranties the MMSE criterion. However it still is the best trade-off between performance and computational load (Edfors, 1996). 


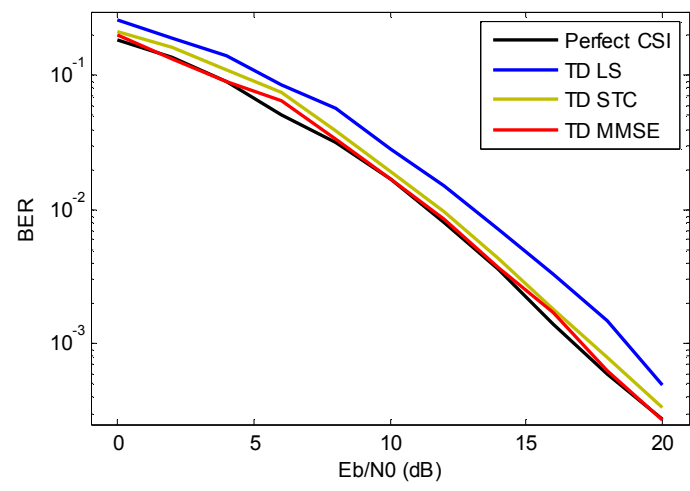

Figure 3: System BER performance (BRAN-A indoor channel).

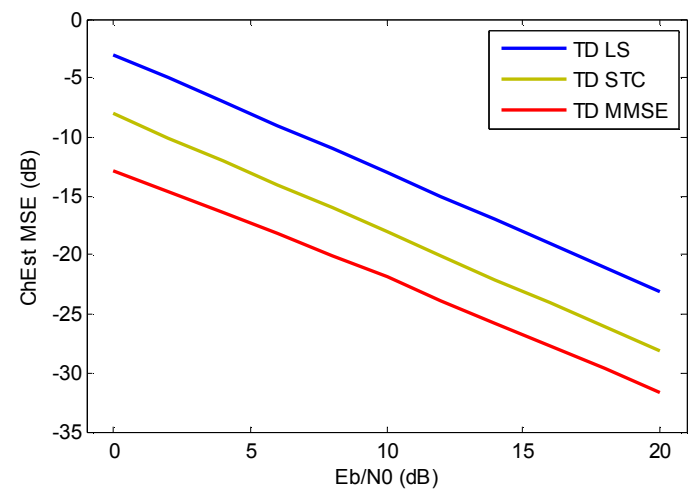

Figure 4: Channel estimation MSE (BRAN-A indoor channel).

\section{SIMULATION RESULTS}

A simulation scenario was implemented using an Alamouti $2 \times 1 \quad$ OFDM system with $N c=1024$ QPSK modulated sub-carriers, sampling interval $\Delta t=10 n s$ and a CP with 200 samples. The transmitted OFDM symbols carried pilots and data using the proposed pilot structure, with a pilot separation $N f=4$.

The BRAN-A and BRAN-E channel models [14] were used to simulate indoor (50ns rms delay spread) and outdoor environments (250ns rms delay spread), respectively.

To validate the proposed method, BER and channel estimation simulations were performed, using $\mathrm{Eb} / \mathrm{N} 0$ values in the range of $0 \mathrm{~dB}$ to $20 \mathrm{~dB}$. The 3 channel estimation schemes presented in section 3 were simulated with both channel models.

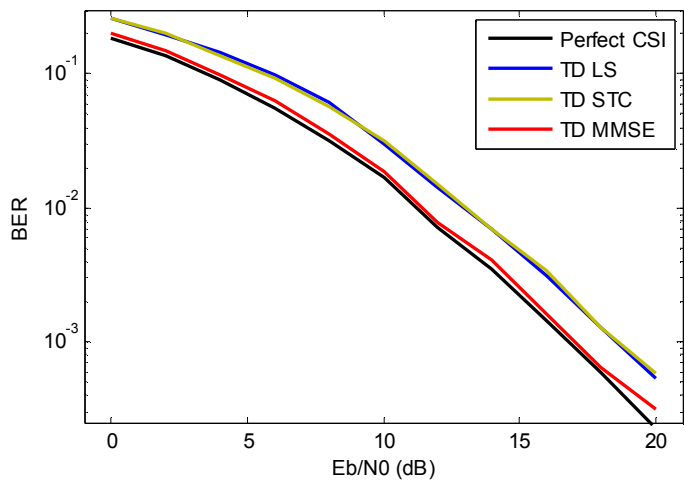

Figure 5: System BER performance (BRAN-E outdoor channel).

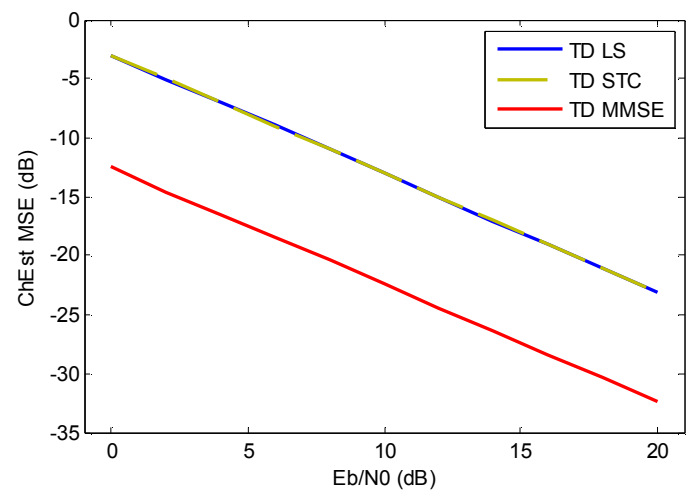

Figure 6: Channel estimation MSE (BRAN-E outdoor channel).

The BER results are depicted in Figure 3 and Figure 5, respectively for BRAN-A and BRAN-E channels.

The normalized channel estimation MSE results are depicted in Figure 4 and Figure 6, respectively for BRAN-A and BRAN-E channels.

On both scenarios, the TD LS and the TD MMSE methods present a consistent performance. The TD LS method always achieves the worst performance $(\approx 2 d B$ degradation in the BER compared with the perfect channel state information - CSI) due to the fact that it does not take advantage of the channel characteristics. In opposition, the TD MMSE method always achieves the best performance, near the ideal situation. It has the ability of dealing with the increasing channel delay spread by always weighing the energy of channel taps vs. noise variance.

The performance of the TD STC method ( $\mathrm{Li}$, 2002) is closely dependent on the channel delay spread. Its performance is bond by the 2 previous methods. A channel with a short delay spread will 
result in the best performance (by having the CIR energy concentrated to just a few taps, most of the noise is eliminated in the estimation process). As the channel delay spread increases, the performance tends to that of the TD LS method, with a significant performance degradation.

\section{CONCLUSIONS}

We have presented a pilot sequence design and associated channel estimation algorithms for MIMOOFDM systems, where all transmit antennas share the same sub-carriers to convey pilot symbols. The proposed design grants the receiver the ability of extracting the CIRs by processing the antennas' TD received samples, without co-channel interference, resulting in an algorithm with very low computational load.

The investigated method presents a considerable performance improvement when compared to the method in (Li, 2002), especially in the more demanding outdoor environments, where it shows the ability of maintaining the performance despite the increased channel delay spread.

\section{ACKNOWLEDGEMENTS}

The authors wish to thank Fundação para a Ciência e a Tecnologia that partially supported this work through the project "PHOTON - Distributed and Extendible Heterogeneous Radio Architectures using Fibre Optic Networks" (PTDC/EEATEL/72890/2006).

\section{REFERENCES}

Nee, R. V., Prasad, R., 2000. OFDM for Wireless Multimedia Communications, Artech House. $1^{\text {st }}$ edition.

Stuber, G. L., Barry, J. R., McLaughlin, S. W., Li, Y., Ingram, M. A., Pratt, T. G., 2004. Broadband MIMO-
OFDM wireless communications. In Proceedings of the IEEE, vol. 92, no. 2, pp. 271-294.

Sampath, H., Talwar, S., Tellado, J., Erceg, V., Paulraj, A., 2005. A fourth-generation MIMO-OFDM broadband wireless system design, performance, and field trial results. In IEEE Communications Magazine, pp. 143-149.

Paulraj, A. J., Gore, D. A., Nabar, R. U., Bolcskei, H., 2004. An overview of MIMO communications - a key to gigabit wireless. In Proceedings of the IEEE, vol. 92, no. 2, pp. 198-218.

Li, Y. G., Seshadri, N., Ariyavisitakul, S., 1999. Channel estimation for OFDM systems with transmitter diversity in mobile wireless channels. In IEEE Journal on Selected Areas in Communications, vol. 17, no. 3, pp. 461-471.

Li, Y. G., 2002. Simplified channel estimation for OFDM systems with multiple transmit antennas. In IEEE Transactions on Wireless Communications, vol. 1, pp. $67-75$

Shin, M., Lee, H., Lee, C., 2004. Enhanced channelestimation technique for MIMO-OFDM systems. In IEEE Transactions on Vehicular Technology, vol. 53, no. 1 , pp. 261-265.

Zhang, H., Li, Y. G., Reid, A., Terry, J., 2005. Channel estimation for MIMO-OFDM in correlated fading channels. In IEEE International Conference on Communications, pp. 2626-2630.

Zamiri-Jafarian, H., Pasupathy, S., 2007. Robust and improved channel estimation algorithm for MIMOOFDM systems. In IEEE Transactions on Wireless Communications, vol. 6, pp. 2106-2113.

Barhumi, I., Leus, G., Moonen, M., 2003. Optimal training design for MIMO OFDM systems in mobile wireless channels. In IEEE Transactions on Signal Processing, vol. 51, pp. 1615-1624.

Minn, H., Al-Dhahir, N., 2004. Optimal training signals for MIMO OFDM channel estimation. In IEEE Global Telecommunications Conference, pp. 219-224.

Ribeiro, C., Gameiro, A., 2007. Direct time-domain channel impulse response estimation for OFDM-based systems. In IEEE Vehicular Technology Conference.

Edfors, O., 1996. Low-complexity algorithms in digital receivers, Ph.D. dissertation, Lulea University of Technology, Sweden.

ETSI TS 101475 V1.2.2, 2001. Broadband Radio Access Networks (BRAN): HIPERLAN Type 2: Technical specification; Physical layer. 